\title{
Discussion on the Fusion and Evolution of Bodiless Lacquerware Workmanship and Contemporary Sculpture*
}

\author{
Jiamao Liu \\ Fine Arts and Design College \\ Minjiang University \\ Fuzhou, China 350000
}

\begin{abstract}
Although the lacquer and sculpture are two different art forms of art, they have a close relationship. Through the organic fusion of Fuzhou Bodiless Lacquerware workmanship and sculpture modeling, will make the art of "Lacquer sculpture"send out unique artistic charm.
\end{abstract}

Keywords—fusion; characteristics; Lacquer sculpture; color

\section{INTRODUCTION}

Fuzhou Bodiless Lacquerware is an art treasure with unique national style and rich local characteristics, it and Beijing's cloisonne, Jiangxi Jingdezhen porcelain are known as the "Three treasures" of traditional Chinese crafts and renowned at home and abroad, its superb technology, smooth and flawless, its gloss like mirror and reflect people clearly, its carcass is thin and colorful, its gold and silver are fabulous and eye-catching. Fuzhou Bodiless Lacquerware by absorbing a variety of ancient Chinese painting cultures, gradually grow up in Qianlong years of the Qing Dynasty, and enter its heyday of development at the end of Qing Dynasty. Its variety is very rich, such as the large lacquer painting screen displayed in the Great Hall of People, bodiless antique bronze lion, painted large vases, tea sets, cans, bowls, dishes, plates, etc., total nearly a thousand varieties.

\section{THE CHARACTERISTICS OF FUZHOU BODILESS LACQUERWARE WORKMANSHIP}

Fuzhou Shen Shaoan's bodiless lacquerware is the most representative flag of Chinese folk lacquerware since Qianlong years of Qing Dynasty. Shen Shaoan fully recovered our long lost traditional bodiless lacquerware skills, and his descendants on the basis of inheritance of its superb skills, also created thin material lacquer workmanship, it produced thin material lacquer through grinding gold and silver foil into mud and pour into the dry lacquer, and then use the thumb dip thin material and slap on the lacquer surface, this method greatly saved the using amount of lacquer, but also produced an unexpected artistic effect. Since then, Fuzhou lacquerware gradually formed the artistic characteristics which is good at bodiless lacquerware, light and solid, colorful, bright and rich in

*Young teachers education and research project of Fujian Provincial Department of Education, project number: JAS160413. modeling .The lacquer art master Li Zhiqing who return from Japan combined Japan's coating process and China's filling technology together and explored a whole set of thick filling technique. Fuzhou lacquer practitioners summarized it as "Qicai piaobian", "Qili xisha", "Shanguang chenhua", "Tiexiu tongban", "Xipi qizhou", "Xibo qiansi", "Binglie sheduan" seven techniques

\section{The PRESENTED ARTISTIC APPEARANCE AFTER THE FUSION OF FUZHOU BODILESS LACQUERWARE AND CONTEMPORARY SCULPTURE}

There are many similarities between contemporary sculptures and bodiless lacquerware, they generally presented in three-dimensional shapes, and the post effect processing of contemporary sculptures are often painted with lacquers. Also they all use some mechanisms to carry out artistic expression. If combined Fuzhou bodiless lacquerware skills into the sculpture modeling, so that it has a lacquerware as light and gorgeous, rich texture, brilliant effect, while playing the advantages of sculpture modeling, the final formed "Lacquer sculpture" will have a strong visual impact and appeal, while combined creative ideas and new performance techniques of contemporary art into the works, lacquer sculpture will show its unique artistic effect.

Many of Fuzhou bodiless lacquerware art crafts themselves are three-dimensional modeling presented as sculpture art, those two has each other inside themselves, facing such huge artistic wealth, we use what we learned, combined contemporary sculpture art concept into it, and created lacquer sculpture with distinctive character, which is the local sculptor's historical responsibility and mission.

In recent years, there are individual sculptors trying to conduct lacquer craft effect processing on the sculpture surface, but paint the surface of the lacquer effect processing, but barely works. It is because most of the sculptors did not know well about the lacquer craft. Coupled with the complex process of lacquer, you need to invest a lot of time to do lacquer effect processing. In order to make a deep integration of lacquer art and sculpture, you must have a deep understanding of the lacquer craft, especially as Fuzhou bodiless lacquerware craft with deep background and superb skills, we need to spend a lot 
of time to get familiar and control its production process and performance techniques.

\section{THE MOLDING FEATURE OF LACQUER SCULPTURE}

Lacquer sculpture modeling is different from the plane lacquer painting modeling, it is a solid form exists in the threedimensional space, the audience can watch and play at all angles, but as a two-dimensional lacquer painting art can only be viewed in a specific angle, and is not suitable to play with and intimate contact. As a three-dimensional modeling, the lacquer sculpture has more intense visual impact and artistic appeal.

Although lacquer sculpture and the traditional Fuzhou Bodiless Lacquerware all presented in three-dimensional shape but there is a big difference with the performance theme and modeling style. The performance theme of lacquer sculpture is mainly about the characters, animals and other specific things, the performance theme of Fuzhou bodiless lacquerware is more about practical life utensil modeling. For modeling style, lacquer sculpture modeling is relatively more complex, Fuzhou bodiless lacquerware modeling is more simple and pure.

\section{EMBRYo BODY MATERIAL OF LACQUER SCULPTURE}

There are many kinds of embryo body materials, such as: wood embryo, metal embryo, stone embryo, synthetic resin embryo, ceramic embryo and so on. Different embryonic body materials have different properties.

\section{A. Linen Embryo}

Linen embryo, linen is the most commonly shaping material used in Fuzhou bodiless lacquerware, also the embryo body material of lacquer sculpture can made with linen, linen sculpture embryo body is solid and light with strong plasticity, it is a very good material. Linen has a very strong tension, use lacquer to stick the linen on the body of plaster mold, and then brush the lacquer mud on the linen net, after three or four layers it will form a certain thickness. Please note that when you stick the linen you should use fine linen on the place where close to lacquer layer, burlap usually used under the fine linen. Burlap generally use sacks cloth, grass linen, fine linen often use gauze, silk cloth, bean bag cloth and so on. The used cloth should have a certain thickness with thick cloth eyes, cloth material should be pure natural.

\section{B. Wood Embryo}

Wood embryo body is light and Eco-friendly, it give a sense of intimacy, and when you hold it in hand it is not that cold. At the same time lacquer and wood could also form a perfect combination which could withstand the erosion of time and could keep the lacquer for a long time. A lot of the Warring States period lacquerware could be preserved until now is a good evidence for that. But the wood material is not easy to process, also the lacquer sculpture produced by highquality wood has high cost. On wood embryo it should apply ash scraping and lacquer painting, so the wood embryo body should be as thin as possible.

\section{Synthetic Resin Embryo}

Synthetic resin material is a common material used in sculptures in recent years, this material is easy to process and with low cost, now there are many artists try to make lacquer effect processing on synthetic resin, and the effect is also good. This material is easy to mold and suitable for mass production.

\section{Metal Embryo}

There are many works which apply lacquer effect processing on metal materials. Metal material is hard and solid, so it can withstand the invasion of wind and rain, compared with other embryo bodies, its stability will be better. But there are some notions for the lacquer effect processing on metal material, the surface of the metal should not be too smooth, so it can be roughly polished by 400 \# waterproof abrasive paper, then brush a layer of raw lacquer and put into the oven, baking it with the temperature between $120{ }^{\circ} \mathrm{C} \sim 160{ }^{\circ} \mathrm{C}$ in order to enhance the adhesion of lacquer. The lacquer should cover the entire surface of metal material so that it is completely wrapped with lacquer, which can make better resistance of the penetration and erosion of rain.

\section{E. Stone Embryo}

Sculpture with stone embryo body can also apply lacquer on the surface, but the stone surface should not be too smooth, or else the lacquer will be easy to peel off. Also it can be roughly polished by 400 \# waterproof abrasive paper, then brush a layer of raw lacquer and put into the oven to bake in order to enhance the adhesion of lacquer.

\section{F. Ceramic Embryo}

Ceramics is the quintessence of China, after thousands of years of accumulation, ceramic culture has penetrated into the hearts of the vast majority of Chinese people. Apply the lacquer effect processing on ceramic sculpture surface has always been attempted by many artists. Taiwan's lacquer artist Mr. Huang Baoxian did a deep research in this area, and achieved fruitful results. On the surface of rough pottery we can apply lacquer effect processing directly on it, but if make lacquer effect processing on the smooth ceramic surface, especially on the bright glazed surface, it is necessary to carry out the special treatment, we need to make sandblasting treatment on the bright ceramic surface, and then brush a layer of varnish on it, next make low-temperature baking at the temperature between $120{ }^{\circ} \mathrm{C} \sim 160{ }^{\circ} \mathrm{C}$, so that the lacquer can be firmly attached to the ceramic surface. Apply lacquer effect treatment on ceramic sculpture surface can be divided into local painting and overall painting two forms, local painting can highlight the diversity of its artistic performance, but also retains the artistic charm of ceramics. The overall painting is mainly use ceramic embryo body for artistic creation, it emphasizes the artistic characteristics of lacquer and its visual experience.

\section{THE COLOR PROCESSING ON LACQUER SCULPTURE SURFACE}

The surface of the sculpture is usually colored in the post production stage. The color processing is directly related to the 
success or failure of the sculpture creation. The color of the lacquer sculpture is greatly enriched by the expression of the lacquer craft. Because of the importance of color, the color processing of sculpture modeling surface needs careful thinking, the lacquer effect processing of lacquer sculpture should also consider the visual expression of the modeling. Normally the color should not be too fancy in order to deal with the delicate balance between modeling and color, if the color is too garish then it will directly effect the artistic expression of sculpture modeling, if the color is too monotonous, then it will make the overall vision of the sculpture lacks vitality. We need to make different lacquer effect processing based on different lacquer sculpture modeling. The color and texture of simple shaped lacquer sculpture can be rich, thus highlighting the artistic expression of lacquer art; the color and texture of complex modeling lacquer sculpture should not be too rich and fancy.

The material of sculpture itself has its own color attributes, when we make the color processing for lacquer sculpture, we should also respect the color of the material, such as the wood itself has a natural color and texture, most of the wood can bring different degrees of kind, warm feelings to people, so when brush on wood sculpture, it will be appropriate to retain some of the natural texture of wood. Lacquer matches with gold, silver and other metal materials, lacquer's gloss and metal's gloss could blend together very well.

Red and black are the most representative colors of Fuzhou bodiless lacquerware. The red lacquer is the loudest red in the red, the black lacquer is the most beautiful black, the combination of the two natural lacquers is loud and stable, and as time goes on, the two colors have become the symbol of lacquer art. Lacquer sculpture should also inherit these cultural characteristics in color processing, so that the lacquer cultural language of sculpture could be more prominent.

\section{PRODUCTION PROCESS OF LACQUER SCULPTURE}

The production method of lacquer sculpture is to combine the sculpture forming method and the production method of Fuzhou bodiless lacquerware. The early stage is mainly the production of sculpture manuscript and the preparation of the outer mold, later stage is to make body and lacquer decoration. Work "Zuo Yi" "Fig. 1" is a lacquer sculpture work through the combination of Fuzhou bodiless lacquerware craft and sculpture, the specific production process is as follows:

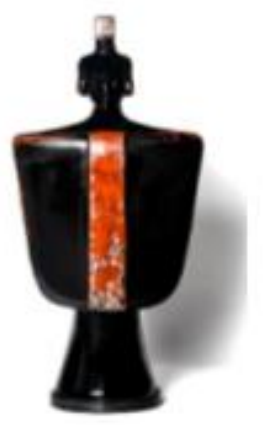

Fig. 1. Work "Zuo Yi"
- Create a clay small draft of "Zuo Yi", determine the specific size of the sculpture.

- Enlarge the size and make the manuscript according to the size of the sculpture

- Use gypsum to remake the outer mold of the draft, open the mold, remove the soil in the mold, clean the gypsum mold, dry in the air.

- Evenly brush a layer of release agent in the gypsum, soap or floor wax could be the release agent.

- Apply one layer of lacquer mud, placed in the shade to dry, simple polished

- First brush a layer of fine ash, and then brush a layer of medium ash, then brush a layer of rough ash, placed in the shade room to dry, simple polished

- Use lacquer to paste a thin cloth, and then mounted three layers of linen, each layer should brush a layer of lacquer mud to fill the mesh of the cloth.

- Brush fine ash, medium ash, rough ash each one layer, placed into the shade room to dry.

- Brush one layer of raw lacquer, placed into the shade room to dry, polished.

- Soak in the water, use tools to open the gypsum mold, remove the character body shape.

- Brush a layer of lacquer on the body and then brush a layer of raw lacquer, polished.

- Brush a layer of khaki colored lacquer on the head of the figure and the clothes, then brush a layer of red lacquer, placed in the shade room to dry, polished with 800 \# waterproof abrasive paper.

- Brush a layer of black polished lacquer, placed into the shade room to dry, polished with 1000 \# waterproof abrasive paper, complete the lacquer sculpture work.

The final "cover lacquer", "grinding lacquer", "push light" process of lacquer sculpture are very important processes, the thickness of "cover lacquer" should be moderate, the grinding force should be just right, and only in this way could create the ideal lacquer sculpture work.

\section{CONCLUSION}

The natural fusion of Fuzhou bodiless lacquerware workmanship and contemporary sculpture makes the lacquer sculpture sent out a unique artistic charm, through color, texture, gloss and other visual language make the audience have a simple elegant, or gentle soft, or rich and luxurious artistic beauty. The blend of the two forms of art makes the sculptor and lacquer artist's works more diversified and personalized, the modeling of the work becomes more beautiful, the color of the work becomes richer, and the performance techniques of the work are more diversified. I believe that in the near future, lacquer sculpture art will definitely usher in the development of its spring. 


\section{REFERENCES}

[1] Yan Limin: On the integration and evolution of modern lacquer and sculpture [J], Arts and Crafts, 1st issue in 2015, P. 44-45.

[2] She Guohua, Chen Qinqun: Creation, sculpture and space - limited outlook of contemporary lacquer art [J], Sculpture, 4th issue in 2014, P. 56-57.

[3] Li Jinxian: Encounter of sculpture and lacquer art- lacquer performance of flower sculpture series [J], Sculpture, 1st issue in 2012. P. 54-55.

[4] Xu Jing: The lacquer context in decorative sculpture [J], Art, 211th issue in 2009, P. 187

[5] Zhu Chonghua: Lacquer and art [M], Shenyang: Liaoning Fine Arts Publishing Company, 2008, P. 65-66.

[6] Bi Guoqin: Lacquer design [M], Shanghai: Shanghai Pictorial Publishing Company, 2007, P.42-43. 